\title{
MONITORING AND ANALYSIS OF TEA ROOT PARAMETERS BASED ON CI600 IN SITU ROOT IMAGER
}

\author{
Sun, D. Z. $.^{1,2,3,4}-$ WANG, W. X.1,3,4* - HUANG, Y ${ }^{5^{*}}-$ SonG, SH. R. ${ }^{1,2,3,4}-$ XIE, J. X. $.^{1,2,3,4}-$ \\ LAI, J. G. ${ }^{1}-$ PENG, G. ${ }^{1}$

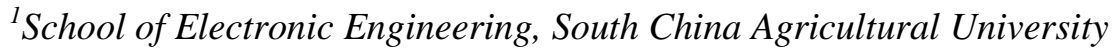 \\ Guangzhou 510642, China \\ ${ }^{2}$ Guangdong Citrus Mango Technology System Machinery Research Laboratory \\ Guangzhou 510642, China \\ ${ }^{3}$ Guangdong Agricultural Information Monitoring Engineering Technology Research Center \\ Guangzhou 5106424, China \\ ${ }^{4}$ Guangzhou Key Laboratory of Agricultural Information Acquisition and Application \\ Guangzhou 510642, China \\ ${ }^{5}$ Liuzhou Railway Vocational Technical College, Liuzhou Guangxi 545616, China \\ ${ }^{*}$ Corresponding authors \\ e-mail: weixing@scau.edu.cn (Wang,W.X.); huangying800816@163.com (Huang, Y.)
}

(Received $8^{\text {th }}$ Jul 2019; accepted $16^{\text {th }}$ Oct 2019)

\begin{abstract}
Four groups of one-year-old Yinghong No. 9 tea plants were studied under with different gradients of water. The correlation test and analysis of soil moisture and tea root coefficient, root length, root area and root volume were carried out. The number, length, area and the volume of tea roots were collected by CI600 plant root growth monitoring equipment, and the correlation between the parameters and water was analyzed to determine the suitable range of soil moisture for tea plant growth. The results showed that the soil moisture suitable for tea tree growth in the case of red loam soil was $35 \%$. During the 90-day test period, 13 young roots were newly born for tea trees with $35 \%$ soil moisture, the root length increased by $299.02 \mathrm{~mm}$, the root area increased by $1285.207 \mathrm{~mm}^{2}$, and the volume increased by $1169.063 \mathrm{~mm}^{3}$. The number, length, area and volume of roots were positively correlated with the growth time at soil moisture of $35 \%$, and the $\mathrm{R}^{2}$ values were $0.9887,0.9907,0.9801$ and 0.9919 , which were higher than those under difference soil moisture. At the end of the experiment, the root coefficient of tea roots under $35 \%$ soil moisture increased by 2.16 times; the length of roots increased by 1.0068 times; the area increased by 0.515 times and the volume increased by 0.64 times. The performance of each index of tea planted under $25 \%$ soil moisture was slightly worse than for those tea planted under $35 \%$ soil moisture. The performance of each index of tea planted under $15 \%$ and $45 \%$ soil moisture was average. So the number, length, area and volume of tea roots are positively correlated with growth time at soil moisture of $35 \%$. The tea tree under other moisture conditions does not exhibit this relationship.
\end{abstract}

Keywords: $C 1600$, tea tree, root monitoring, soil moisture, situ root measurements

\section{Introduction}

Root system is an important way of material exchange between plants and their environment, and the primary means of water intake. Root system can absorb a large amount of water from soil and provide life activities (Hu, 2013; Guo, 2018). The growth and development of plants are reflected by root growth state, which is mainly concentrated in the development and distribution of roots and physiological characteristics (Wang, 2007; Wang, 2015; Niu, 2017). 
Different morphology of root growth is showed under different water stress conditions. Studies have shown that plants subjected to water stress will adapt to water deficit by changing root structure and morphology, which had higher plasticity (Zhanyang, 2017). Zhu (2012) and Lai (2018) presented that the morphological structure and root biomass of the root system may change accordingly to the change of soil moisture, which affected the physiological characteristics of root system and the growth and development of the whole plant. Zhou (2008) pointed out that the root growth index of citrus increased with the increase of water, and Liu (2014) pointed out that the activity and osmotic adjustment ability of various enzymes in roots of droughtresistant peanut varieties were higher than those of weak drought-resistant varieties. Wei (2011) concluded that there is a significant correlation between root volume and water in jujube trees in horizontal and vertical directions. Meng et al. (2017) said that the development of root hair is regulated by a variety of nutrient elements.

The analysis and research methods of root system have made great progress and development from the initial soil-removing research to the later digital analysis, but there are still some limitations, such as inconvenience monitoring methods and complexity of real-time analysis (Liu, 2013; Qin, 2019). The root growth state under water stress was monitored by CI600 plant root growth. The number, length, area and volume of tea roots were collected. The data were analyzed by least squares method to obtain the correlation between root related parameters and soil moisture. Relationships provide a reference for the scientific planting of tea trees.

The article aimed to use CI600 plant root growth monitoring equipment to analyze the roots of four years old Yinghong No. 9 tea plants under varying soil moisture, in order to find the optimal value.

\section{Materials and Method}

\section{Test environment}

Experiment location: The experimental site is located in the campus of South China Agricultural University, Guangzhou City, Guangdong Province (11334'N, 2314'E). The region is warm and rainy, with humid climate and abundant sunshine. It belongs to the subtropical monsoon climate. The annual average temperature is around 20 to $22^{\circ} \mathrm{C}$; the annual rainfall is $1720 \mathrm{~mm}$, and the annual relative humidity is $77 \%$. Good climatic conditions are suitable for most plants. The experiment lasted from Feb.10 to May 11, 2019; the daily average maximum temperature was $25^{\circ} \mathrm{C}$; the daily average minimum temperature was $17^{\circ} \mathrm{C}$, and the daily average relative humidity was $53 \%$, which is suitable for plant growth and control of experimental variables.

\section{Plant culture}

Four groups of one-year-old Yinghong No. 9 tea trees with the same growth potential and smaller root differences were selected for experiment. The tea roots need to be properly trimmed to make the whole suitable for the test requirements, and the treated tea trees are planted in a soil box containing red loam for 20 days. Only 2 one-year-old tea trees are planted for each of soil moisture content. 


\section{Experiment equipment}

(1) DECAGON Em50 Data Logger (DECAGON, USA), including sensors and collectors, is used to collect soil moisture.

(2) CI600 root parameters monitoring system (CID-Bioscience, USA), includes CI600 Root Scanne (Hardware Scanner), CI600 In-Situ Root Imager (Scanning Software) and CI690 Root Snap (Image Analysis Software).

(3) SHT11 air temperature and humidity sensor is used to measure the temperature and humidity of the air.

\section{Experimental design}

Four kinds of soil moisture were designed, which were $15 \%, 25 \%, 35 \%$ and $45 \%$. The red loam soil was mixed, stirred evenly, and sand and gravel were removed before being packed. The weight was evenly distributed into four soil tanks and compacted. The average weight of red loam in each soil box was $20 \mathrm{~kg}$. The experimental tea tree is shown in Figure 1.
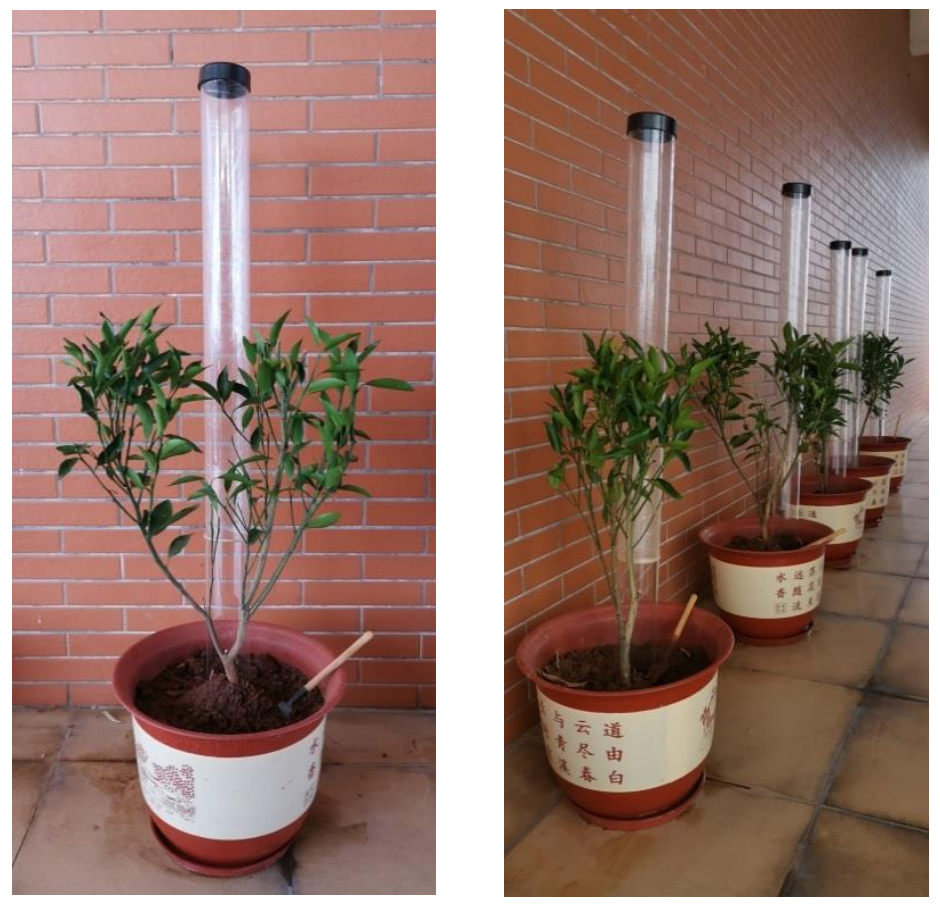

Figure 1. The experimental tea tree

\section{Experiment results and analysis}

The root coefficient, root length, root area and root volume of the tea tree roots are measured by CI600 Root Scanne and CI600 In-Situ Root Imager. Date will be automatically calculated by CI690 RootSnap. Resultes is processed by averaging its data.

The root coefficient is defined as the number of tea roots that grow during the test period.

Root coefficient, root length, root area and root volume of tea trees will be tested to find out the effect and relationship of soil water content between them. 
The growth rate is defined as the ratio of the current root coefficient to the previous root coefficient.

The growth rate is given by Equation (1).

$$
\text { Growth } \text { rate }=\frac{N_{2}-N_{1}}{N_{1}} * 100 \%
$$

where $\mathrm{N}_{2}$ represents the parameter measured at the moment, and $\mathrm{N}_{1}$ represents the parameter measured at the previous moment. These parameters can be root coefficient, root length, root area and root volume of tea trees.

\section{Effect of Soil Moisture on Root Coefficient of Tea Trees}

The variation of the root coefficient of tea tree under different soil moisture is shown by CI600 Root Scanne and CI600 In-Situ Root Imager, and the corresponding curve is shown in Figure 2.

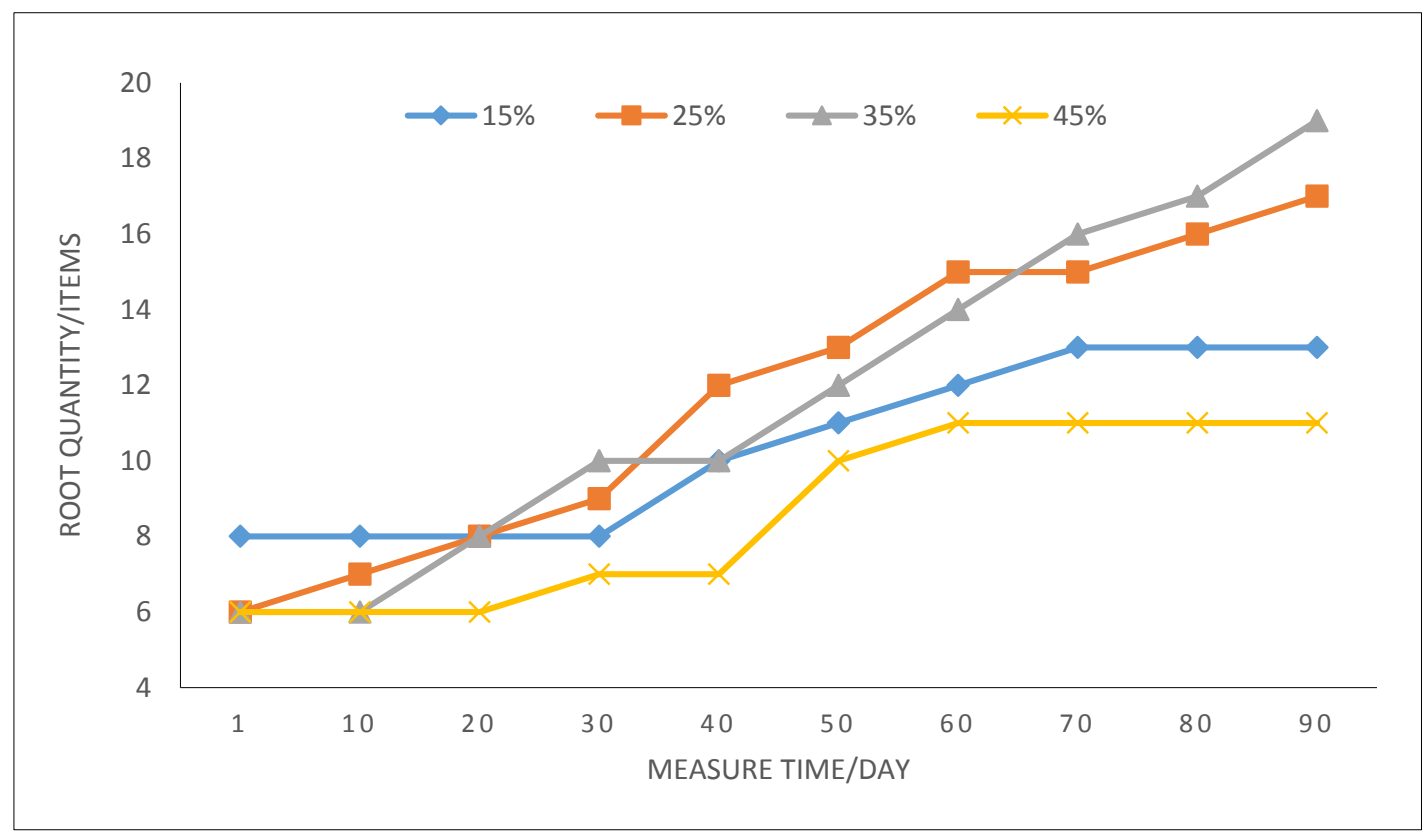

Figure 2. Curve of the variation of tea root coefficient under different soil moisture

Experiments show that the coefficient of tea root system under four soil moisture conditions increased by $5,11,13$ and 5 , and increased by $0.625,1.83,2.16$ and 0.83 times. Figure 2 shows that the rate of increase of root coefficient of tea tree planted under $35 \%$ soil moisture was relatively stable, and had a good performance but slowed in the later stage under $25 \%$ soil moisture. Compared with $35 \%$ soil moisture, root coefficient growth rate fluctuates too much throughout during the experiment period. However, the root coefficient of tea trees planted under 15\% and $45 \%$ soil moisture has almost no change. It can be speculated that this humidity is not suitable for tea tree growth. The relationship between the four root coefficient quantities and the growth time was curve-fitted, and the determination coefficients $\mathrm{R}^{2}$ were $0.9108,0.9773$, 0.9887 and 0.8685 . 


\section{Effects of Soil Moisture on Root Length of Tea Trees}

The root length of tea tree under different soil moisture is measured by CI600 Root Scanne and CI600 In-Situ Root Imager., and the corresponding curve is shown in Figure 3.

Experiments show that the initial root length of tea planted with $15 \%$ soil moisture is significantly longer than that of the other three groups. It can be seen from Figure 3 that the root lengths of tea trees planted under $15 \%$ and $45 \%$ soil moisture have similar trends, while the root lengths of tea trees planted under $25 \%$ and $35 \%$ soil moisture are similar, but the growth rate of root length is significantly larger than that. $15 \%$ and $45 \%$ soil moisture in two groups of tea trees. Compared with the beginning of the experiment, the root length of tea planted under four soil moisture conditions increased by $31 \%, 83.5 \%, 100.68 \%$ and $37.75 \%$ at the end of the experiment. It can be speculated that it is more suitable for tea tree root growth under the $35 \%$ soil moisture. The relationship between the four root coefficient quantities and the growth time was curvefitted, and the determination coefficients $\mathrm{R}^{2}$ were $0.9711,0.9938,0.9907$ and 0.9763 .

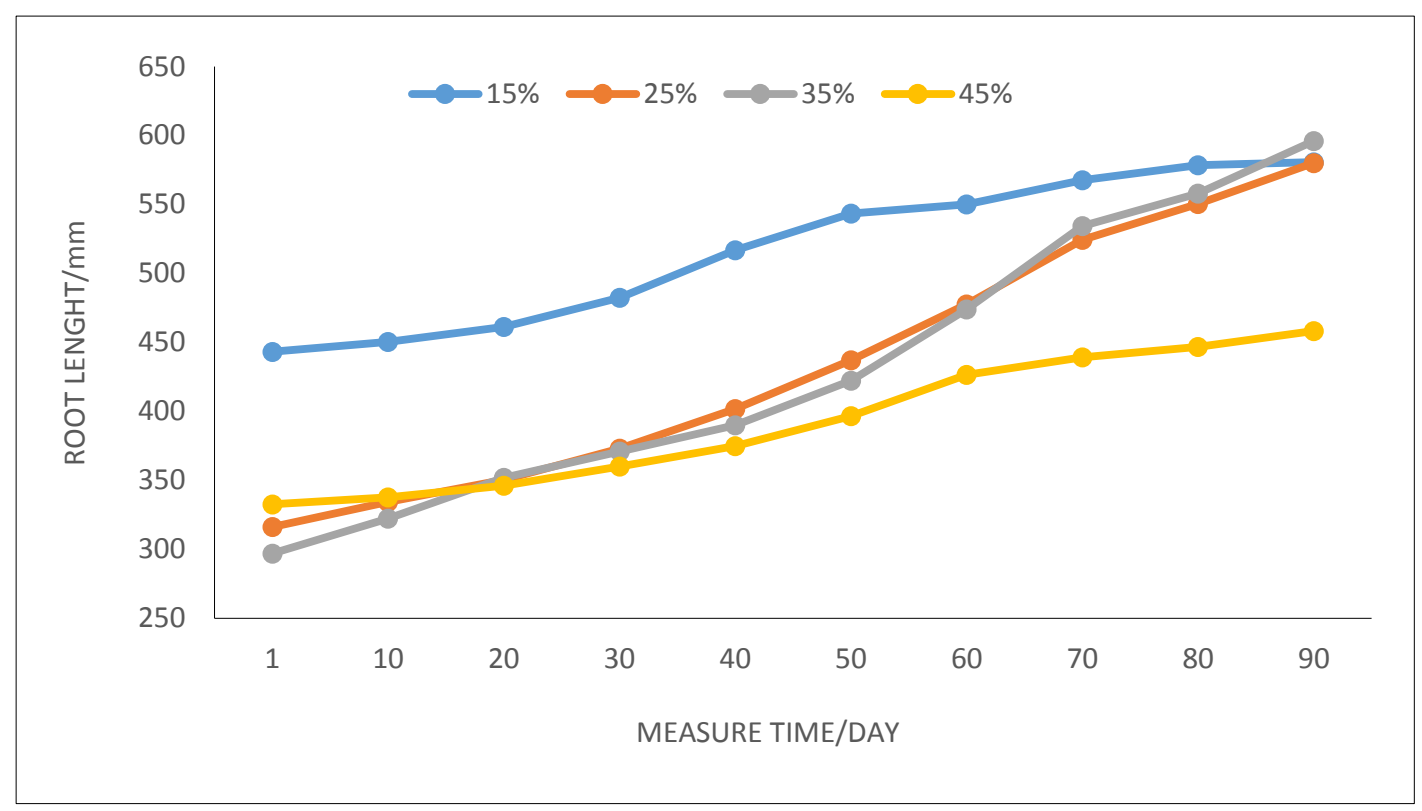

Figure 3. Curve of the variation of tea root length under different soil moisture

\section{Effect of Soil Moisture on Root Area of Tea Trees}

The changes of root area of tea planted under different soil moisture were obtained from experimental calculations and the corresponding curves were shown in Figure 4.

Experiments show that the root area of tea tree planted under four soil moisture conditions increased by $259.509 \mathrm{~mm} 2,879.491 \mathrm{~mm} 2,1285.207 \mathrm{~mm} 2$ and 369.107 $\mathrm{mm} 2$, with corresponding growth rates of $7.56 \%, 33.4 \%, 51.5 \%$ and $13.6 \%$. Figure 4 shows that the root area of tea trees planted under $15 \%$ and $45 \%$ soil moisture is relatively flat, which may be caused by more serious water stress. The root area of tea trees planted under $25 \%$ soil moisture increased rapidly, and the tea trees planted at $35 \%$ soil moisture increased fastest, indicating that root growth was the fastest. The determination coefficients $\mathrm{R}^{2}$ were $0.9867,0.9938,0.9801$ and 0.9946. 


\section{Effects of Soil Moisture on Root Volume of Tea Trees}

The change of tea root area under different soil moisture was obtained from experimental calculations and the corresponding curve was shown in Figure 5.

Experiments show that the initial value of root volume of tea tree planted under $15 \%$ soil moisture is larger. During the experimental period, the root area of tea planted under 4 kinds of soil moisture increased by $214.458 \mathrm{~mm}^{3}, 766.661 \mathrm{~mm}^{3}, 1169.063 \mathrm{~mm}^{3}$ and $316.605 \mathrm{~mm}^{3}$, with corresponding growth rates of $8.8 \%, 40.2 \%, 64.0 \%$ and $15.8 \%$. Figure 5 shows that the root volume of tea trees planted under $15 \%$ and $45 \%$ soil moisture increased more slowly, while the root volume of tea planted under $25 \%$ and $35 \%$ soil moisture increased more rapidly. The tea tree planted under $35 \%$ soil moisture increased the fastest, reaching $64 \%$, indicating that the root growth was the fastest. The determination coefficients $\mathrm{R}^{2}$ were $0.9957,0.9957,0.9919$ and 0.9869 , respectively.

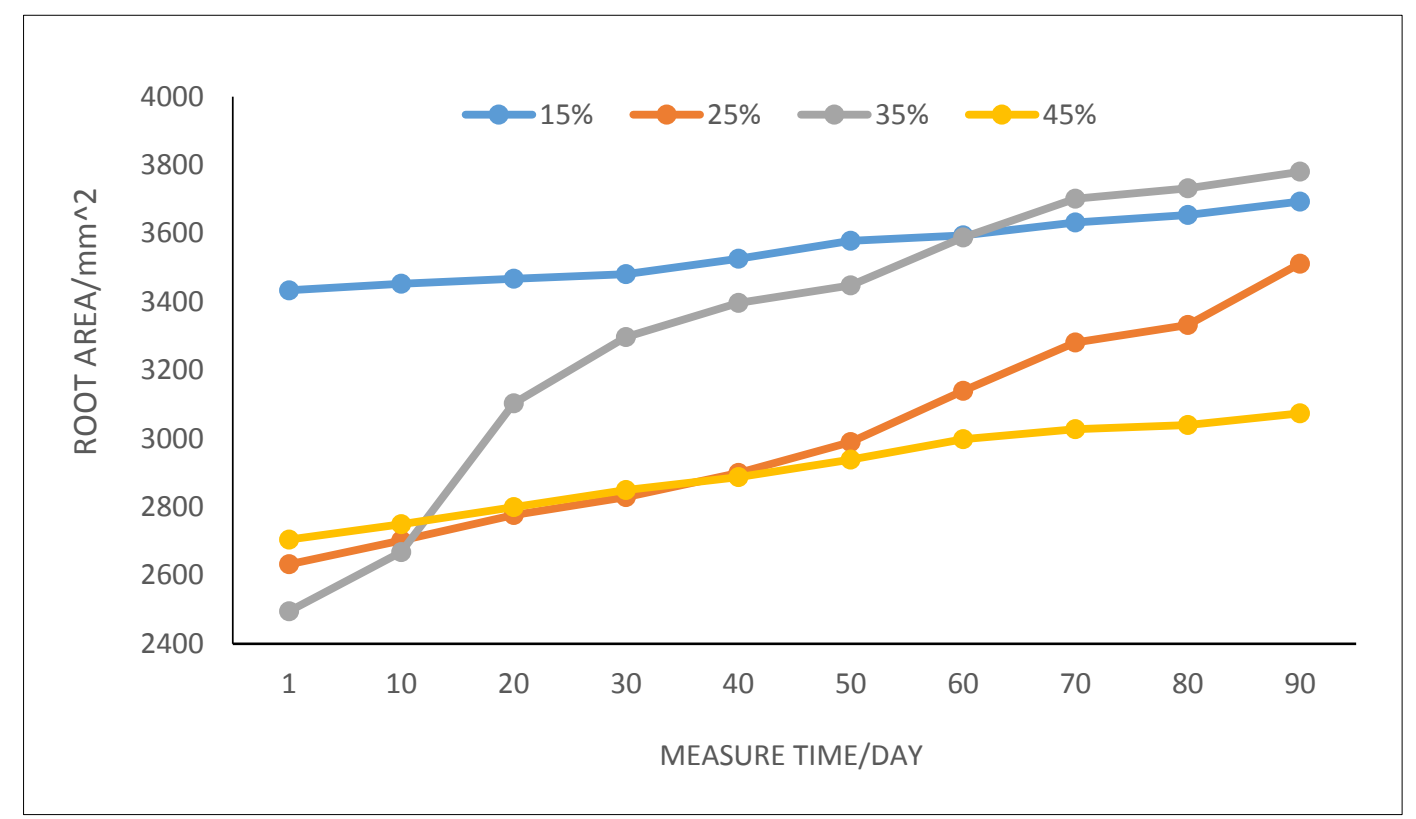

Figure 4. Curve of the variation of tea root area under different soil moisture

The comparison of various indicators of tea roots planted under four kinds of soil moisture is shown in Figure 6.

Figure 6 shows that the growth rate of various roots of tea trees planted under the condition of soil moisture of $35 \%$ is higher than that of other tea trees, and the growth rate of $25 \%$ is slightly worse. Soil moisture of $15 \%$ and $45 \%$ soil moisture is not conducive to the roots growth of tea trees.

\section{Conclusions}

First, the relationship between the root development of tea plants and soil moisture was studied. The changes of the number, length, area and volume of tea roots under different soil moisture were studied. Experiments were studied out with 4 groups of one-year-old Yinghong 9 tea trees. The soil moisture was collected by DECAGON 
Em50 Data Logger, and the related indicators of tea roots were monitored by CI600 root growth monitoring system.

Second, the results show that the growth rate of various roots of tea trees planted under the condition of soil moisture of $35 \%$ is higher than that of other tea trees, and the growth rate of $25 \%$ is slightly worse. Soil moisture of $15 \%$ and $45 \%$ soil moisture is not conducive to the roots growth of tea trees. The number, length, area and volume of tea roots are positively correlated with growth time at soil moisture of $35 \%$. The tea tree under other moisture conditions does not exhibit this relationship. This conclusion can be seen in the experiment and analysis in the second chapter of the paper.

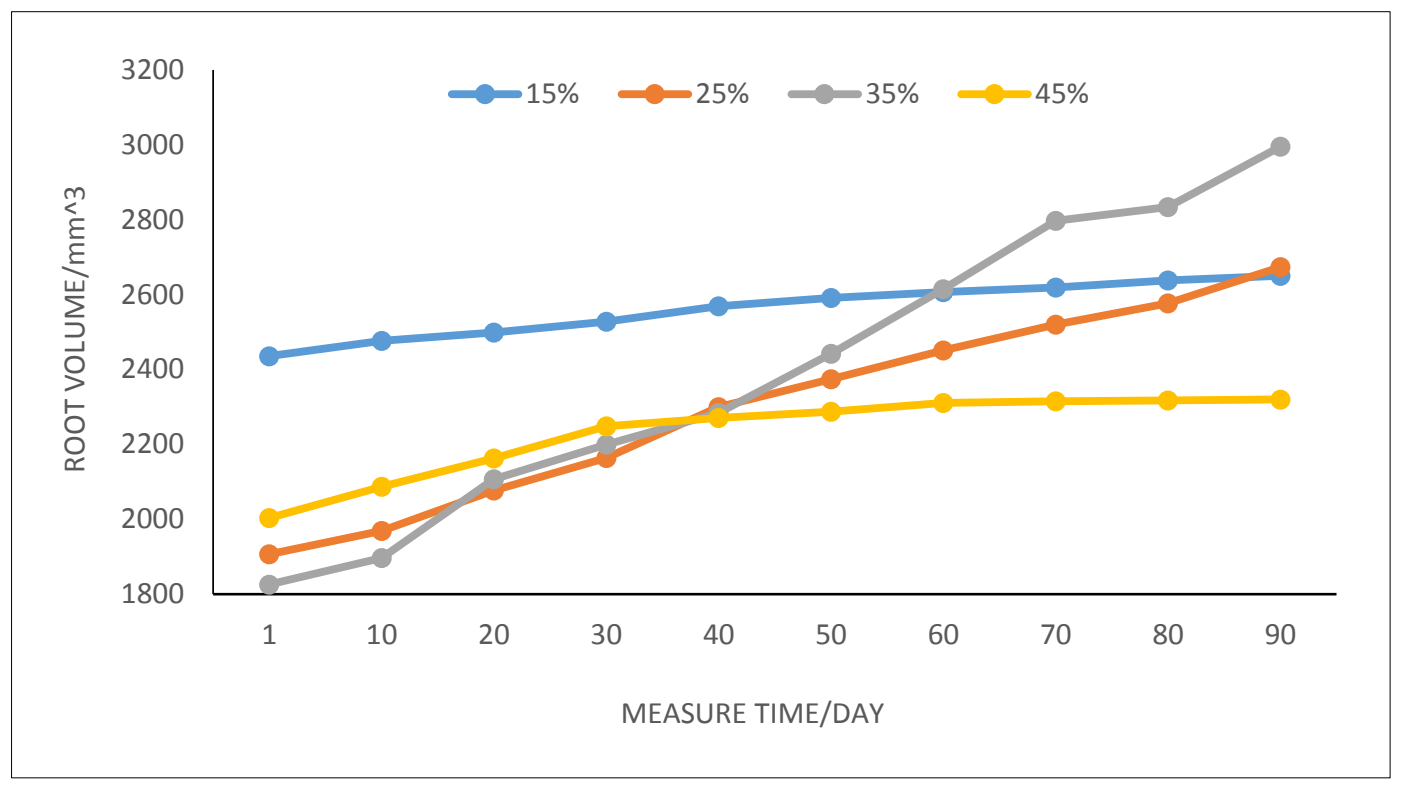

Figure 5. Curve of the variation of tea root volume under different soil moisture

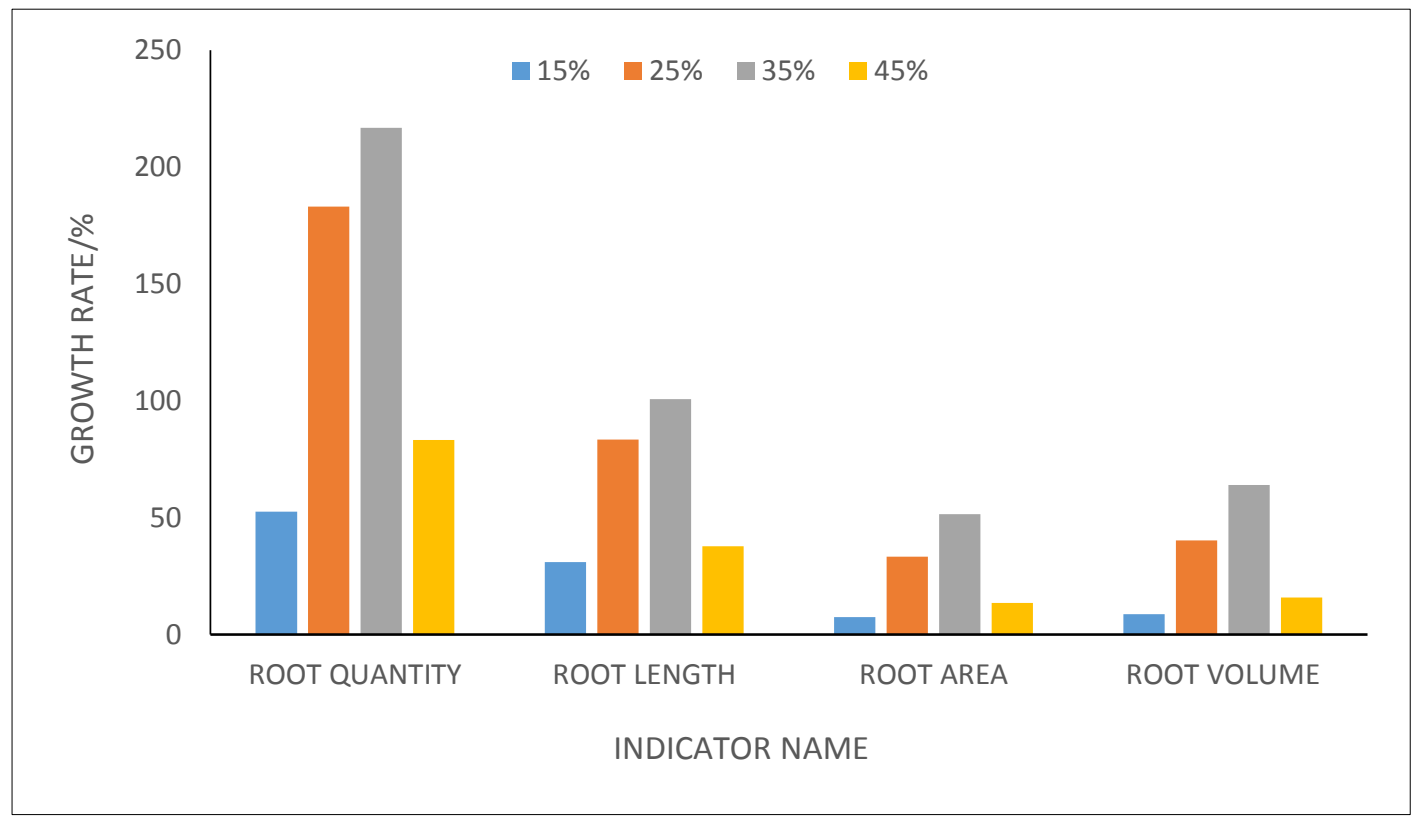

Figure 6. Contrast of the growth rate of each index of tea root system 


\section{Discussion}

The root surface and root volume are derived measures therefore analysing both of those is needed further discussion. The preliminary tests were not considered adequately, and subsequent tests will focus on the parameters that the instrument can directly measure.

The container capacity has been taken into account during the test. The roots of adult tea trees are more developed, and the $20 \mathrm{~L}$ container obviously does not meet the requirements. However, the root system of one-year old tea tree is relatively small. According to the experimental observation, the $20 \mathrm{~L}$ container does not affect the root development. In the later experiments, the tea tree has been transplanted to cuboid container with a length, width and height of $60 \mathrm{~cm} * 60 \mathrm{~cm} * 55 \mathrm{~cm}$.

The test period is only 90 days, and it is carried out in the spring. The growth period is too short and the weather conditions are relatively simple. Subsequent repeated tests will prolong the test time and improve the credibility of the measured data.

The red loam soil was mixed, stirred evenly, and sand and gravel were removed before being packed. However, the nutrient and porosity characteristics of the soil were not analyzed. The experiment only considered the influence of soil moisture on root development. The factors such as nutrient, illumination, air temperature and humidity were not taken into consideration, and subsequent multi-factor tests were carried out. The analysis had significant effects on the root development.

Only four kinds of soil moisture were designed. It was roughly concluded that tea root system developed better when soil moisture was 35\%. However, but no further subdivision and tracking test were carried out. The subsequent research will be carried out between soil moisture of $25 \%-35 \%$.

Acknowledgements. The paper is funded by Liuzhou Science and Technology Plan Project (under No. 2017BE10303), Guangxi Science and Technology Key Research and Development Project (under No. Guike AB16380286), Guangdong Province Key Area R\&D Program (under No. 2019B020214003), Guangdong Science and Technology Plan Project (under No. 2017A020208049), 2018 Provincial Agricultural Science and Technology Innovation and Extension Project (under No. 2018LM2163), South China Agricultural University Science and Technology Achievement Transformation and Incubation Project (under No. CGZHT2018004), Guangdong Universities and Universities Characteristic Innovation Project (2018KTS)CX020, 2018 Provincial College Students Innovation and Entrepreneurship Project (under No. 201810564197), Guangdong Science and Technology Project (under No. 2017A020208049), Modern Agricultural Industry Technology System Construction Project (under No. CARS-27), Guangxi High School Innovation Team and Excellence Scholar Program.

\section{REFERENCES}

[1] Guo, B., Dai, Y., Song, L. (2018): Research progress of plant hormones affecting crop root development under drought. - Biotechnology Bulletin 34(7): 48-56.

[2] Hu, H. (2013): Effect of water on germination, seedling growth and natural distribution of Bermudagrass. - Jiangxi: Jiangxi Normal University.

[3] Lai, J., Li, X., Xue, L. (2018): Advances in plant drought resistance. - Jiangsu Agricultural Science 46(17): 23-27.

[4] Liu, X., Liu, L. (2013): An overview of root research methods. - Agricultural Science and Technology Newsletter 3: 147-148. 
[5] Liu, Y. (2014): Study on the relationship between drought resistance of peanut varieties and root morphology, distribution and physiological characteristics. - Shandong: Shandong Agricultural University.

[6] Meng, H., Feng, J., Hu, D. (2017): Root system development and nutrient uptake. Shanxi Agricultural Science 45(6): 1048-1052.

[7] Niu, X., Nanbiao (2017): Progress in the study of fine roots of grassland plants by microroot canal technique. - Journal of Grassland Science 26(11): 205-215.

[8] Qin, T., Sun, C., Bi, Z. (2019): Advances in plant root imaging technology and application prospects of potato root research. - Journal of Nuclear Agriculture 33(2): 0412-0419.

[9] Wang, C., Sun, X., Ma, J. (2007): Study on water uptake by plant roots. - Shanxi Water Conservancy 1: 85-88.

[10] Wang, Q., Rong, L. (2015): Research progress on the growth and distribution characteristics of plant roots under environmental impact. - Journal of Guiyang University 10(4): 61-66.

[11] Wei, G. (2011): Root distribution of jujube trees on sloping land and its relationship with soil moisture and nutrients. - Shaanxi: Northwest University of Agriculture and Forestry Science and Technology.

[12] Zhou, J. (2008): Study on the effect of red soil water condition on Citrus physiological and ecological factors and its mechanism. - Nanjing: Nanjing Agricultural University.

[13] Zhu, Y. (2012): Progress in plant root growth and research methods. - Northern Horticulture 20: 176-179. 